\title{
Multiple microRNAs regulate human FOXP2 gene expression by targeting sequences in its 3' untranslated region
}

\author{
Lijuan $\mathrm{Fu}^{1+}$, Zhimin Shi ${ }^{1+}$, Guanzheng Luo ${ }^{2}$, Weihong Tu${ }^{1}$, XiuJie Wang ${ }^{2}$, Zhide Fang ${ }^{3}$ and XiaoChing $\mathrm{Li}^{{ }^{*}}$
}

\begin{abstract}
Background: Mutations in the human FOXP2 gene cause speech and language impairments. The FOXP2 protein is a transcription factor that regulates the expression of many downstream genes, which may have important roles in nervous system development and function. An adequate amount of functional FOXP2 protein is thought to be critical for the proper development of the neural circuitry underlying speech and language. However, how FOXP2 gene expression is regulated is not clearly understood. The FOXP2 mRNA has an approximately 4-kb-long 3' untranslated region ( $3^{\prime}$ UTR), twice as long as its protein coding region, indicating that FOXP2 can be regulated by microRNAs (miRNAs).
\end{abstract}

Findings: We identified multiple miRNAs that regulate the expression of the human FOXP2 gene using sequence analysis and in vitro cell systems. Focusing on let-7a, miR-9, and miR-129-5p, three brain-enriched miRNAs, we show that these miRNAs regulate human FOXP2 expression in a dosage-dependent manner and target specific sequences in the FOXP2 $3^{\prime}$ UTR. We further show that these three miRNAs are expressed in the cerebellum of the human fetal brain, where FOXP2 is known to be expressed.

Conclusions: Our results reveal novel regulatory functions of the human FOXP2 $3^{\prime}$ UTR sequence and regulatory interactions between multiple miRNAs and the human FOXP2 gene. The expression of let-7a, miR-9, and miR-129-5p in the human fetal cerebellum is consistent with their roles in regulating FOXP2 expression during early cerebellum development. These results suggest that various genetic and environmental factors may contribute to speech and language development and related neural developmental disorders via the miRNA-FOXP2 regulatory network.

Keywords: miRNAs, FOXP2, 3' UTR, Post-transcriptional regulation, Speech and language, Cerebellum

\section{Background}

Dysfunctions of the human FOXP2 gene have been implicated in speech and language impairments [1-6]. As a transcription factor, the FOXP2 protein controls the expression of hundreds of downstream genes, many of which play important roles in nervous system development and function [7-11]. An adequate amount of functional FOXP2 protein is thought to be critical for the proper development of the distributed neural circuits underlying speech and language $[12,13]$. However, how the expression of the human FOXP2 gene is regulated is not clearly understood. miRNAs are

\footnotetext{
* Correspondence: xli4@|suhsc.edu

${ }^{\dagger}$ Equal contributors

${ }^{1}$ Neuroscience Center of Excellence, LSU Health Sciences Center, New Orleans, LA 70112, USA

Full list of author information is available at the end of the article
}

small nonprotein-coding RNA molecules that regulate gene expression post-transcriptionally by targeting specific sequences in the 3 '-UTRs of mRNAs, leading to mRNA degradation and/or translational suppression $[14,15]$. The FOXP2 mRNA has an approximately 4-kb long 3' UTR, twice as long as its protein coding region [1], raising the possibility that FOXP2 expression is regulated by miRNAs. Here we report the identification of multiple miRNAs that downregulate the expression of the human FOXP2 gene by targeting specific sequences in its 3' UTR.

\section{Results}

Identification of miRNA binding sites in the human FOXP2 3' UTR

Using cDNAs made from human fetal brains combined with polymerase chain reaction (PCR), we cloned a 
$3845 \mathrm{nt}$ sequence fragment that matched $100 \%$ to the human FOXP2 3' UTR sequence annotated in the NCBI database. We searched this FOXP2 3' UTR sequence for miRNA binding sites, requiring a perfect match to the 7 -nt seed sequence in a miRNA $[15,16]$. This search predicted numerous miRNAs that potentially target the FOXP2 3' UTR. Some of these miRNAs have more than one binding site and the binding site sequences are conserved in higher vertebrate species. We selected 12 miRNAs: miR-9, miR-19b, miR-27b, miR-92a, miR-140-5p, miR-190, miR-200a, let-7a, miR-129-5p, miR-582-5p, miR892a, and miR-1237 (Figure 1) and tested whether they downregulate FOXP2 expression in cell culture systems.

\section{Multiple miRNAs downregulate human FOXP2 protein and mRNA expression}

We transfected mimics of each candidate miRNA into human HEK293 cells (where FOXP2 is expressed endogenously) and measured FOXP2 protein levels 72 hours later by Western blot analysis. We found that miR-9, miR-19b, miR-140-5p, miR-200a, let-7a, miR-129-5p, miR-582-5p, and miR-892a reduced FOXP2 protein levels significantly (Figure 2A). Of these miRNAs, let-7a, miR-9, and miR129-5p were among the most effective regulators, reducing FOXP2 protein by $70-90 \%$; they are also known to be abundantly expressed in vertebrate brains $[17,18]$. We focused on these three miRNAs and tested whether their regulatory effects were dose-dependent at three dosages: 2 , 6.5, and $20 \mathrm{nM}$. At $2 \mathrm{nM}$, comparing to the control, let7a and miR-9 each decreased FOXP2 protein levels by about 50\%, while miR-129-5p decreased FOXP2 protein by less than $10 \%(p<0.01$ for all). At $20 \mathrm{nM}$, let-7a and miR-9 decreased FOXP2 protein levels by $90 \%$, and miR$129-5 p$ decreased FOXP2 protein by $70 \%(p<0.001$ for all, Figure $2 \mathrm{~B}$ and $\mathrm{C}$ ). The dose-dependent downregulation was significant for each miRNA $(p<0.0018$ for let-7a and miR-9; $p<0.0006$ for miR-129-5p, Jonckheere-Terpstra test). Using quantitative real time PCR (qRT-PCR), we also found that FOXP2 mRNA level was downregulated by let7a, miR-9, and miR-129-5p in similar transfection experiments (Figure 2D).

let-7a, miR-9, and miR-129-5p target specific sequences in the human FOXP2 3' UTR

Next, we tested whether the regulatory effects of candidate miRNAs were mediated by the 3' UTR of the human FOXP2 gene using luciferase reporter assays. We co-transfected miRNA mimics and a luciferase reporter construct containing a wild type FOXP2 3' UTR or no 3' UTR into SH-SY5Y cells, where no endogenous FOXP2 was expressed, and performed luciferase reporter assays. Compared to a control miRNA with scrambled sequence, the eight candidate miRNAs, miR-9, miR-19b, miR-1405p, miR-200a, let-7a, miR-129-5p, miR-582-5p, and miR-892a significantly repressed luciferase activity when co-transfected with a reporter construct containing a wild type FOXP2 3' UTR ( $p<0.01$ for all), but had no effect when co-transfected with a reporter construct containing no FOXP2 3' UTR. In contrast, miR-27b, miR-92a, miR-190, and miR-1237 did not repress luciferase activity whether the reporter constructs contained a FOXP2 3' UTR or not (Figure 3A). These results were consistent with the Western blot results obtained from HEK293 cells as described in Figure 2A, indicating that the regulatory functions of these miRNAs were independent of the cell lines we used.

We focused on let-7a, miR-9, and miR-129-5p and further tested whether their regulatory effects were sequencespecific. Each of these miRNAs has two binding sites in the human FOXP2 3' UTR. For each miRNA, we made three mutant luciferase reporter constructs with sequences in binding site 1 , binding site 2 , and binding sites $1+2 \mathrm{mu}-$ tated respectively by site-directed mutagenesis (Figure $3 \mathrm{~B}$ ). Using luciferase reporter assays, we showed that each of these miRNAs significantly repressed luciferase activity when only one binding site was mutated $(p<0.05$ or

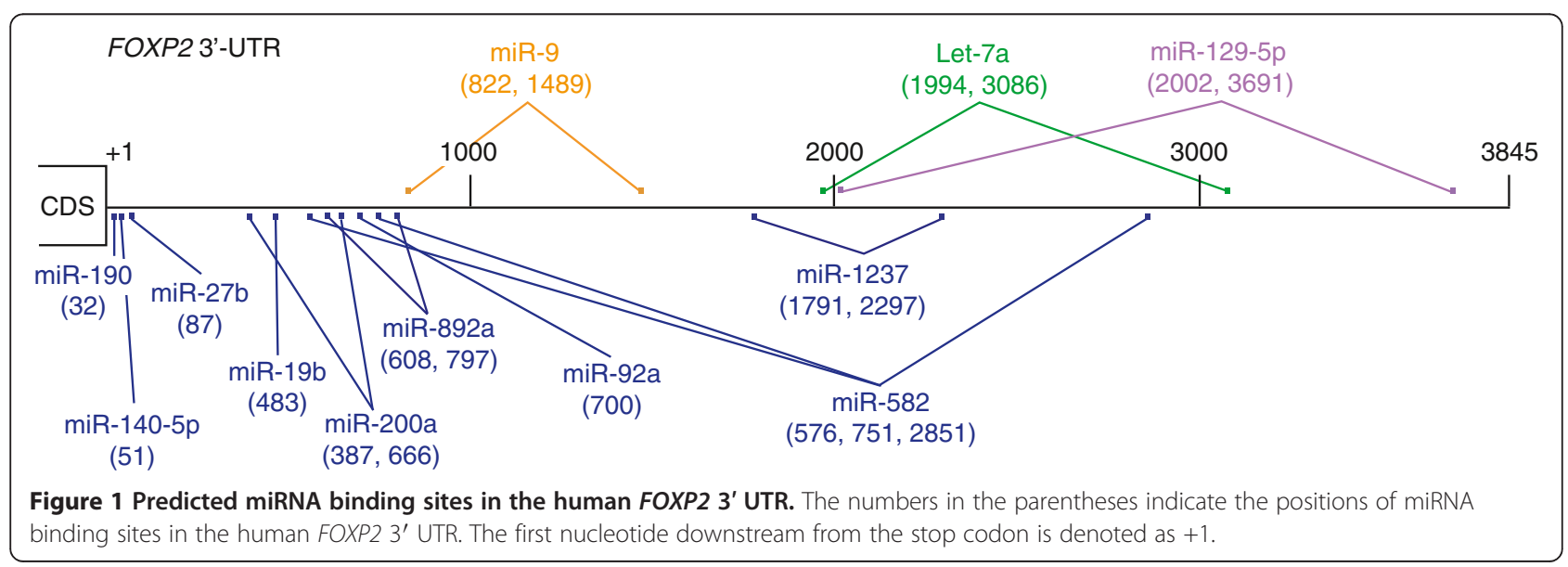




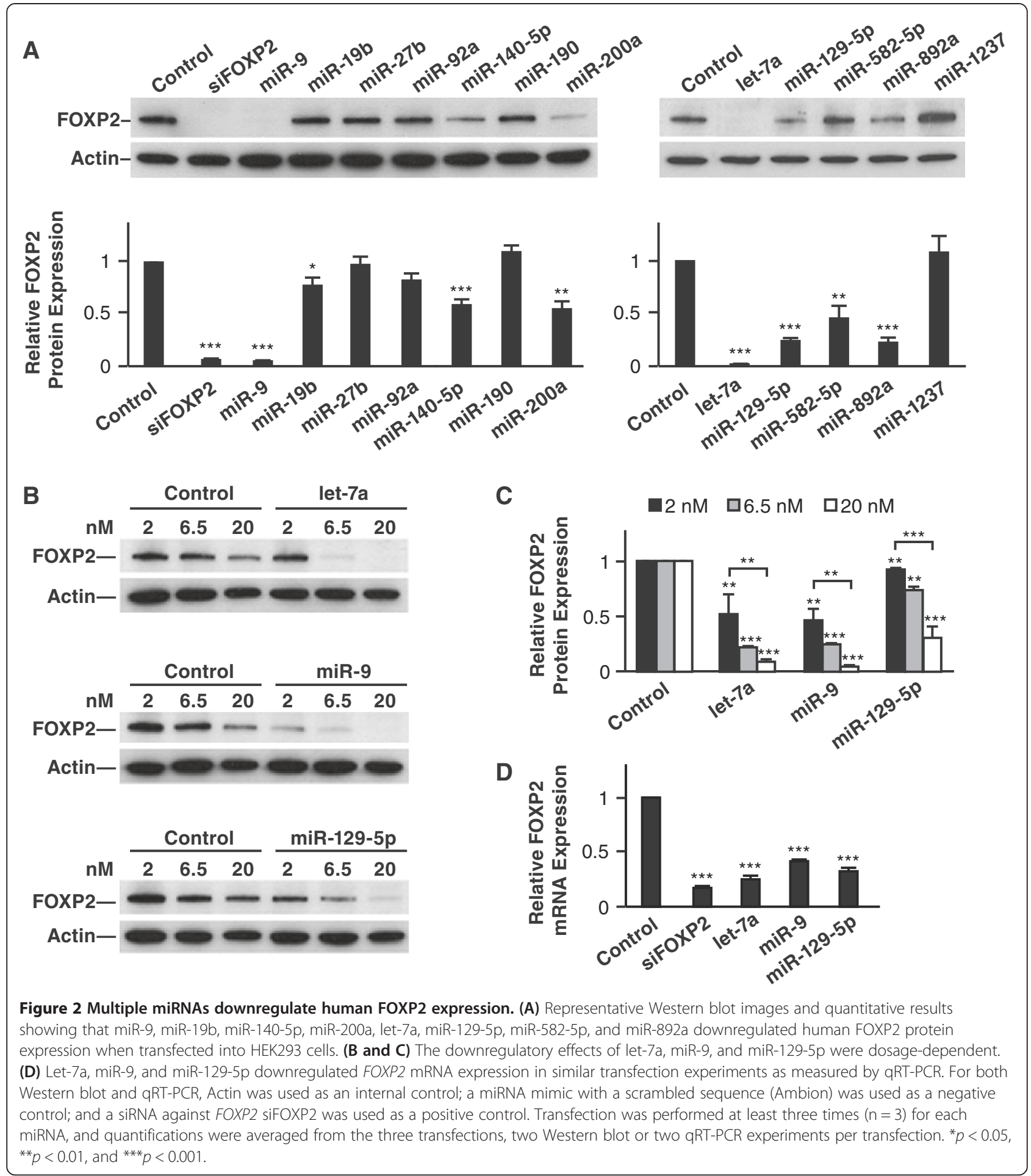

$p<0.01$ for all three miRNAs), but their regulatory effects were completely abolished when both binding sites were mutated ( $p>0.98$ for all three miRNAs, Figure 3C). In comparison, the negative control miRNA had no effect on all reporter constructs whether it had no 3' UTR, wild type 3' UTR, or mutated 3' UTRs (Figure 3C). These results suggested that the downregulatory effects of these miRNAs were mediated through specific sequences in the FOXP2 3' UTR, and each of their respective two binding sites was functional. 


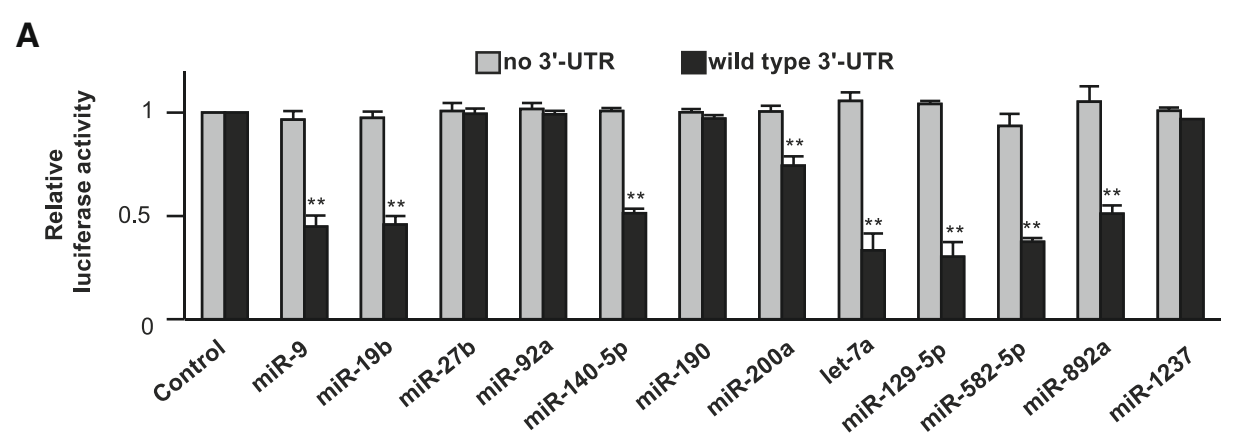

B

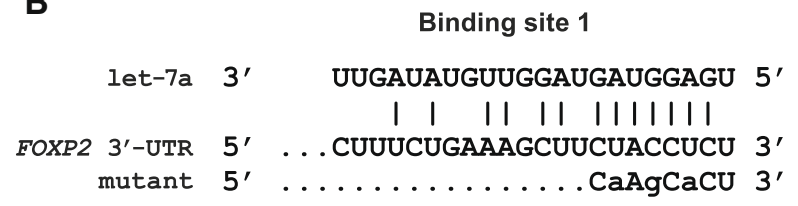

miR-9 $3^{\prime}$ AgUAUGUCGAUCUAUUGgUUUCU 5' FOXP2 $3^{\prime}$-UTR $5^{\prime} \ldots$ AGGCUACAGCUGCAAACCAAAGC $3^{\prime}$ mutant $5^{\prime} \ldots \ldots \ldots \ldots \ldots \ldots$ CCuAAgGC $3^{\prime}$
Binding site 2

3' UUGAUAUGUUGgaUgaUgGagU 5' $5^{\prime}$... UGAAAGAAAACUGUCUACCUCU $3^{\prime}$ $5^{\prime} \ldots \ldots \ldots \ldots \ldots \ldots$ CaAgCaCU $3^{\prime}$

3' AgUaUgucgaucuauUgGUUUCU 5' I I | | |||||| $5^{\prime} \ldots$ UUUAUUUUCAGUCAAACCAAAGU $3^{\prime}$

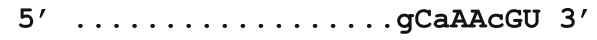

3' CGUUCGGGUCUGGCGUUUUUC 5' I I I | || ||। $5^{\prime} \ldots$ CAUAUCUUACUUAGCAAAAAA $3^{\prime}$ $5^{\prime} \quad \ldots \ldots \ldots \ldots \ldots \ldots \ldots$ g $\ldots \ldots A A A A ~ 3^{\prime}$

C
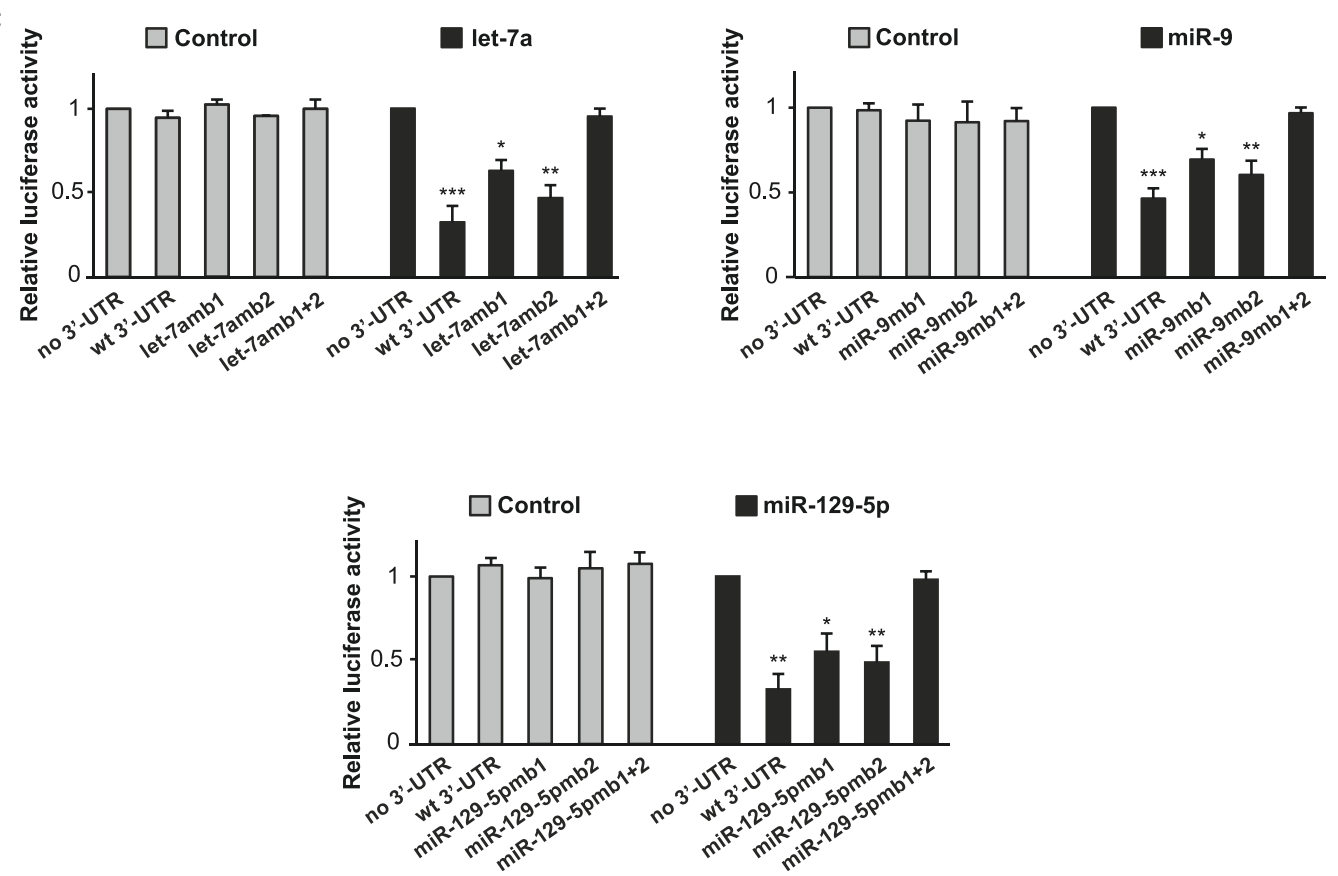

Figure 3 (See legend on next page.) 
(See figure on previous page.)

Figure 3 The downregulatory effects of let-7a, miR-9, and miR-129-5p are mediated via specific sequences in the human FOXP2 3' UTR. (A) miR-9, miR-19b, miR-140-5p, miR-200a, let-7a, miR-129-5p, miR-582-5p, and miR-892a significantly repressed luciferase activity when co-transfected with a reporter construct containing a wild-type FOXP2 3' UTR, but had no effects when co-transfected with a reporter construct containing no $3^{\prime}$ UTR. (B) Mutations in the binding sites for let-7a, miR-9, and miR-129-5p in the human FOXP2 3' UTR. Mutated nucleotides are in lower case letters. (C) Mutations in both the two binding sites for let-7a, miR-9, and miR-129-5p in the FOXP2 3' UTR abolished the repressive effects of these miRNAs in luciferase reporter assays. Transfection was performed three times $(n=3)$ for each miRNA, and luciferase assays were performed in duplicate. To control for transfection efficiency, Renilla luciferase activity was normalized to the internal control firefly luciferase activity. Error bars $=\mathrm{SEM}$; ${ }^{*} p<0.05,{ }^{* *} p<0.01$, and ${ }^{* * *} p<0.001$, Tukey's "Honest Significant Difference" test.

Let-7a, miR-9, and miR-129-5p are expressed in the cerebellum of the human fetal brain

FOXP2 is known to be expressed in several brain regions in the human fetal brain, including the cerebellum, the basal ganglia, the thalamus, and the cortical plate [19]. miRNAs that regulate FOXP2 expression and play a role in brain development are expected to be expressed in similar brain regions. We examined the expression of let-7a, miR-9, and miR-129-5p in human fetal brain tissue by in situ hybridization using Locked Nucleic Acid (LNA) modified miRNA detection probes. Due to limited tissue availability, only the cerebellum (16 weeks old) was examined. We found that all these three miRNAs, let-7a, miR-9, and miR-129-5p, were expressed in the cerebellum of the human fetal brain (Figure 4). These observations were consistent with the roles of these miRNAs in regulating FOXP2 expression during human cerebellum development.
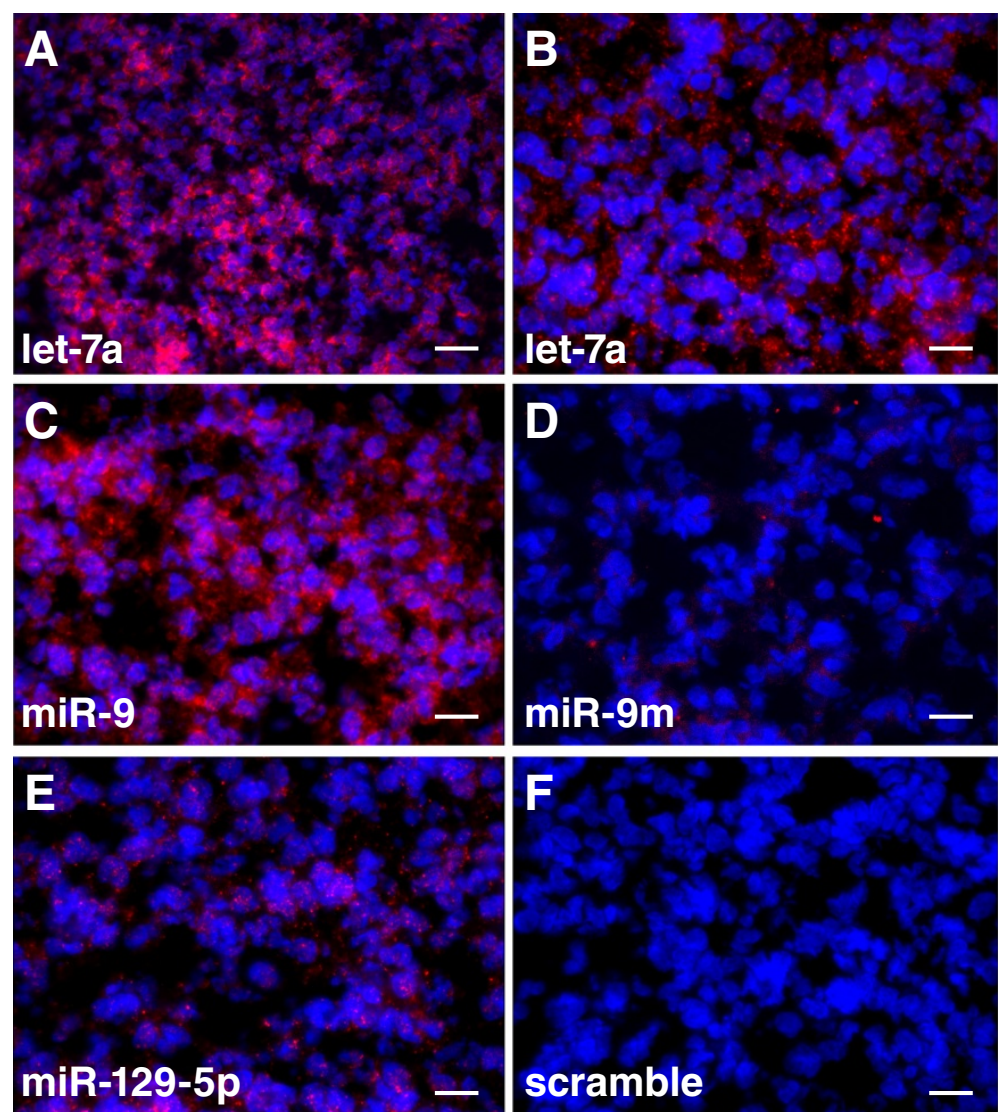

Figure 4 Expression of let-7a, miR-9, and miR-129-5p in the cerebellum of the human fetal brain. In all images, miRNA hybridization signals are in red (Cy3) and DAPI stained cell nuclei are in blue. (A and B) A cerebellum section hybridized with a LNA modified let-7a detection probe. Note the cytoplasmic localization of the let-7a signal. (C) A cerebellum section hybridized with a miR-9 detection probe. (D) A miR-9 probe with 4 nucleotides mutated (miR-9m) detected no hybridization signal. (E) A cerebellum section hybridized with a miR-129-5p detection probe. (F) A negative control probe with scrambled sequence detected no hybridization signal. The scale bars are $40 \mu \mathrm{m}$ in A and $20 \mu \mathrm{m}$ in B through F. 


\section{Discussion}

A heterozygous missense mutation in the human FOXP2 protein coding region (i.e., R553H) results in a transcription factor with compromised DNA binding and transcriptional regulatory activity $[1,20]$. Presumably, reduced functional dosage of cellular FOXP2 protein causes speech and language impairments [12]. Our results, together with recent reports that miR-9, miR-132, and miR-140-5p regulate Foxp2/FoxP2 expression (Foxp2 and FoxP2 denote respective rodent and avian genes) in animal models [21,22], highlight the importance of the FOXP2 3' UTR sequence and the roles for miRNAs in regulating FOXP2 expression. These results raise the possibility that sequence variations in the FOXP2 3' UTR, particularly in miRNA binding sites, due to mutations and/or polymorphism may contribute to dysregulation and/or functional variations of the FOXP2 gene. In addition, various genetic, physiological, and environmental factors may influence the expression of miRNAs, thus indirectly impacting FOXP2 expression.

Human subjects carrying FOXP2 mutations exhibit structural and functional abnormalities, presumably due to FOXP2 malfunction, in several brain regions, including the cerebellum [23], where FOXP2 is known to be expressed [19]. The expression of let-7a, miR-9, and miR-129-5p in the human fetal cerebellum is consistent with their roles in regulating FOXP2 expression during early cerebellum development in humans. Recently, dysregulation of miR-129-5p is found in the cerebellum of autistic brains, albeit only a limited number of brains were examined [24]. Further investigation of in vivo functions of these miRNAs will bring insights into their roles in speech and language development and related neural developmental disorders.

\section{Methods \\ Cloning of the human FOXP2 3' UTR and prediction of miRNA binding sites}

Human fetal brain poly(A)+RNA (Clontech) was reversetranscribed into cDNA using an oligo-dT primer. Using primers designed based on the human FOXP2 3' UTR sequence in the NCBI database [NCBI: NM148898, variant II], we PCR amplified two overlapping fragments representing the FOXP2 3' UTR. The two fragments were ligated at a BamH1 site to obtain a 3845-nt full length human FOXP2 3' UTR. This sequence was used for miRNA binding sites prediction using the software TargetScan. Sequences in the FOXP2 3' UTR matching perfectly to the 7-nt seed sequence of a miRNA were accepted as putative miRNA binding sites [16].

\section{Transfection, Western blotting, and qRT-PCR}

miRNA mimics (Ambion) were transfected into human HEK293 cells $(60 \mathrm{nM})$ using Lipofectamine 2000
(Invitrogen) and cells were harvested 72 hours later for protein and mRNA assays. Proteins were separated by electrophoresis on 10\% SDS-PAGE gel. After blotting, membranes were probed with an antibody against the human FOXP2 protein (sc-21069, Santa Cruz Biotechnology). Protein bands were visualized using ECL-Plus and quantified with Image J. For qRT-PCR, total RNA was extracted from transfected cells and reverse-transcribed (iScript cDNA synthesis kit, Bio-Rad). qRT-PCR was performed using the SYBR Green Supermix (Bio-Rad). Primer sequences are listed in the Additional file 1: Figure S1. The specificity of PCR amplification products were validated by electrophoresis and the melting-curve analysis. For each miRNA, transfection was performed at least three times. For each transfection, Western blot and qRT-PCT were performed at least two times, and qRT-PCR was performed in triplicate.

\section{Luciferase reporter assays}

Plasmid constructs carrying wild-type or mutant human FOXP2 3' UTRs in the psiCHECK vector (Promega, $100 \mathrm{ng})$ were co-transfected with miRNA mimics (100 $\mathrm{nM}$ ) into SH-SY5Y cells. Cells were harvested $48 \mathrm{~h}$ later and luciferase activity was assayed using the DualLuciferase Reporter System (Promega). To control for transfection efficiency, Renilla luciferase activity was normalized to the internal control firefly luciferase activity. For each miRNA, transfection was performed three times and luciferase activity was assayed in duplicate.

\section{Mutagenesis of miRNA binding sites}

The full length human FOXP2 3' UTR sequence was used as a template in site-directed mutagenesis of miRNA binding sites using the QuikChange II XL Mutagenesis Kit (Agilent). Specific PCR primers were designed using the QuikChangePrimer Design tool (see Additional file 1: Figure S1) and synthesized by Integrated Device Technology (IDT: http://www.idt.com). In some cases, to mutate three nucleotides in a binding site, we performed 2 rounds of mutagenesis. For example, we mutated 2 nucleotides in the first round, and used the mutated plasmid as a template in the second round to mutate the third nucleotide. All mutated binding sites were sequence verified.

\section{In situ hybridization}

In situ hybridization was performed as described previously [22]. Briefly, fixed brain sections (10 $\mu \mathrm{m}$ thick) were hybridized with LNA modified miRNA detection probes at $38-45^{\circ} \mathrm{C}$ overnight. After washing and blocking, slides were incubated with an Anti-Digoxigenin antibody for $1 \mathrm{~h}$, followed by signal amplification using the TSA Plus Cy3 System (PerkinElmer). LNA modified miRNA detection probes were purchased from Exiqon: let-7a probe (18000-01); miR-9 probe (88078-05); a 
customer designed mutant miR-9 probe (miR-9m: 5'TCATAGAGCTACATAACCATACA-3', underlined are mutated nucleotides); miR-129-5p probe (38482-15); negative control probe (99004-01). The human fetal brain (16 weeks old) cerebellum sections were obtained from a commercial source (Biochain Institute, CA).

\section{Additional file}

Additional file 1: Figure S1. Sequences of primers used in respective experiments.

\section{Abbreviations}

3' UTRs: 3' untranslated regions; qRT-PCR: Quantitative real time polymerase chain reaction.

\section{Competing interest}

All authors declare that they have no competing interests.

\section{Authors' contributions}

XCL designed the experiment; LF, ZS, WT carried out the experiments and analyzed the results; GZL and XJW contributed to bioinformatics analysis; ZF performed statistical analysis; XCL wrote the manuscript. All authors read and approved the manuscript.

\section{Acknowledgements}

This work was supported by NIH grant (MH081254) and the Brain Behavior Research Foundation's Young Investigator Award to XCL.

\section{Author details}

${ }^{1}$ Neuroscience Center of Excellence, LSU Health Sciences Center, New Orleans, LA 70112, USA. ${ }^{2}$ Key Laboratory of Genetic Network Biology, Institute of Genetics and Developmental Biology, Chinese Academy of Sciences, Beijing 100101, China. ${ }^{3}$ Biostatistics Program, LSU Health Sciences Center, New Orleans, LA 70112, USA.

Received: 8 July 2014 Accepted: 18 September 2014

Published online: 01 October 2014

\section{References}

1. Lai CS, Fisher SE, Hurst JA, Vargha-Khadem F, Monaco AP: A forkheaddomain gene is mutated in a severe speech and language disorder. Nature 2001, 413(6855):519-523.

2. Feuk L, Kalervo A, Lipsanen-Nyman M, Skaug J, Nakabayashi K, Finucane B, Hartung D, Innes M, Kerem B, Nowaczyk MJ, Rivlin J, Roberts W, Senman L, Summers A, Szatmari P, Wong V, Vincent JB, Zeesman S, Osborne LR, Cardy JO, Kere J, Scherer SW, Hannula-Jouppi K: Absence of a paternally inherited FOXP2 gene in developmental verbal dyspraxia. Am J Hum Genet 2006, 79(5):965-972.

3. Lennon PA, Cooper ML, Peiffer DA, Gunderson KL, Patel A, Peters S, Cheung SW, Bacino CA CA: Deletion of 7q31.1 supports involvement of FOXP2 in language impairment: clinical report and review. Am J Med Genet A 2007, 143A(8):791-798.

4. Shriberg LD, Ballard KJ, Tomblin JB, Duffy JR, Odell KH, Williams CA: Speech, prosody, and voice characteristics of a mother and daughter with a 7;13 translocation affecting FOXP2. J Speech Lang Hear Res 2006, 49(3):500-525.

5. Zeesman S, Nowaczyk MJ, Teshima I, Roberts W, Cardy JO, Brian J, Senman L, Feuk L, Osborne LR, Scherer SW: Speech and language impairment and oromotor dyspraxia due to deletion of $7 q 31$ that involves FOXP2. Am J Med Genet A 2006, 140(5):509-514

6. MacDermot KD, Bonora E, Sykes N, Coupe AM, Lai CS, Vernes SC, Vargha-Khadem F, McKenzie F, Smith RL, Monaco AP, Fisher SE: Identification of FOXP2 truncation as a novel cause of developmental speech and language deficits. Am J Hum Genet 2005, 76(6):1074-1080.

7. Shu W, Yang H, Zhang L, Lu MM, Morrisey EE: Characterization of a new subfamily of winged-helix/forkhead (Fox) genes that are expressed in the lung and act as transcriptional repressors. J Biol Chem 2001, 276(29):27488-27497.
8. Spiteri E, Konopka G, Coppola G, Bomar J, Oldham M, Ou J, Vernes SC, Fisher SE, Ren B, Geschwind DH: Identification of the transcriptional targets of FOXP2, a gene linked to speech and language, in developing human brain. Am J Hum Genet 2007, 81(6):1144-1157.

9. Vernes SC, Oliver PL, Spiteri E, Lockstone HE, Puliyadi R, Taylor JM, Ho J, Mombereau C, Brewer A, Lowy E, Nicod J, Groszer M, Baban D, Sahgal N, Cazier JB, Ragoussis J, Davies KE, Geschwind DH, Fisher SE: Foxp2 regulates gene networks implicated in neurite outgrowth in the developing brain. PLoS Genet 2011, 7(7):e1002145.

10. Vernes SC, Spiteri E, Nicod J, Groszer M, Taylor JM, Davies KE, Geschwind DH, Fisher SE: High-throughput analysis of promoter occupancy reveals direct neural targets of FOXP2, a gene mutated in speech and language disorders. Am J Hum Genet 2007, 81(6):1232-1250.

11. Konopka G, Bomar JM, Winden K, Coppola G, Jonsson ZO, Gao F, Peng S, Preuss TM, Wohlschlegel JA, Geschwind DH: Human-specific transcriptional regulation of CNS development genes by FOXP2. Nature 2009, 462(7270):213-217.

12. Fisher SE, Scharff C: FOXP2 as a molecular window into speech and language. Trends Genet 2009, 25(4):166-177.

13. Graham SA, Fisher SE: Decoding the genetics of speech and language. Curr Opin Neurobiol 2013, 23(1):43-51.

14. Lee RC, Feinbaum RL, Ambros V: The C. elegans heterochronic gene lin-4 encodes small RNAs with antisense complementarity to lin-14. Cell 1993 75(5):843-854.

15. Bartel DP: MicroRNAs: target recognition and regulatory functions. Cell 2009, 136(2):215-233.

16. Friedman RC, Farh KK, Burge CB, Bartel DP: Most mammalian mRNAs are conserved targets of microRNAs. Genome Res 2009, 19(1):92-105.

17. Landgraf $P$, Rusu M, Sheridan R, Sewer A, lovino N, Aravin A, Pfeffer S, Rice A, Kamphorst AO, Landthaler M, Lin C, Socci ND, Hermida L, Fulci V, Chiaretti S, Foa R, Schliwka J, Fuchs U, Novosel A, Muller RU, Schermer B, Bissels U, Inman J, Phan Q, Chien M, Weir DB, Choksi R, De Vita G, Frezzetti $\mathrm{D}$, Trompeter $\mathrm{HI}$, et al: A mammalian microRNA expression atlas based on small RNA library sequencing. Cell 2007, 129(7):1401-1414.

18. Pena JT, Sohn-Lee C, Rouhanifard SH, Ludwig J, Hafner M, Mihailovic A, Lim C, Holoch D, Berninger P, Zavolan M, Tuschl T: miRNA in situ hybridization in formaldehyde and EDC-fixed tissues. Nat Methods 2009, 6(2):139-141.

19. Lai CS, Gerrelli D, Monaco AP, Fisher SE, Copp AJ: FOXP2 expression during brain development coincides with adult sites of pathology in a severe speech and language disorder. Brain 2003, 126(Pt 11):2455-2462.

20. Vernes SC, Nicod J, Elahi FM, Coventry JA, Kenny N, Coupe AM, Bird LE, Davies KE, Fisher SE: Functional genetic analysis of mutations implicated in a human speech and language disorder. Hum Mol Genet 2006, 15(21):3154-3167.

21. Clovis YM, Enard W, Marinaro F, Huttner WB, De Pietri Tonelli D: Convergent repression of Foxp2 3'UTR by miR-9 and miR-132 in embryonic mouse neocortex: implications for radial migration of neurons. Development 2012, 139(18):3332-3342.

22. Shi Z, Luo G, Fu L, Fang Z, Wang X, Li X: miR-9 and miR-140-5p target FoxP2 and are regulated as a function of the social context of singing behavior in zebra finches. J Neurosci 2013, 33(42):16510-16521.

23. Watkins KE, Vargha-Khadem F, Ashburner J, Passingham RE, Connelly A, Friston KJ, Frackowiak RS, Mishkin M, Gadian DG: MRI analysis of an inherited speech and language disorder: structural brain abnormalities. Brain 2002, 125(Pt 3):465-478.

24. Abu-Elneel K, Liu T, Gazzaniga FS, Nishimura Y, Wall DP, Geschwind DH, Lao K, Kosik KS: Heterogeneous dysregulation of microRNAs across the autism spectrum. Neurogenetics 2008, 9(3):153-161.

doi:10.1186/s13041-014-0071-0

Cite this article as: Fu et al:: Multiple microRNAs regulate human FOXP2 gene expression by targeting sequences in its $3^{\prime}$ untranslated region. Molecular Brain 2014 7:71 